\title{
Multiple left subclavian venous catheterizations in premature infant
}

\author{
Hyun Soo Moon, Soo Kyung Lee, Eun Young Kim, Manho Kim, and Jung-Min Lee \\ Department of Anesthesiology and Pain Medicine, Hallym University College of Medicine, Anyang, Korea
}

Central venous catheterization (CVC) has been essential in the management of critically ill premature infants for total parenteral nutrition (TPN), hemodynamic monitoring, administration of fluids, drugs or blood products, the route for blood sampling, and etc. [1]. Due to technical difficulties and various complications, a reliable CVC has become an important field of long-term care for low birth weight infants. Complications during and after CVC are divided into three groups: mechanical, thrombotic and infectious complications that make long-term patency impossible in critically ill infants [2]. The puncture techniques, insertion sites, and managements of indwelling central venous catheters are also key factors of long-term patency of CVC. We report a case of three times successful CVC, using the same left subclavian vein in an extremely premature infants with necrotizing enterocolitis and giant hydrocephalus for about three months period, without any remarkable complications.

A premature female infant, weighing $1.28 \mathrm{~kg}$ was transferred from the local clinic, where the infant was delivered by a cesarean section at pregnant 29 weeks gestation. When transferred, she was intubated with self respiration for the management of acute respiratory distress syndrome. Two days after admission, the ventriculargaleal shunt was performed for accompanying giant hydrocephalus, under general anesthesia. After induction of anesthesia, the supraclavicular subclavian venous catheterization (SCSVC) was done at the request of the department of pediatrics. For the CVC of the infant, only the left subclavian vein was available, because the umbilical vein and the both femoral veins were obstructed or unavailable due to previous uses. Furthermore, the right central veins were to be secured by the surgeon for the protection of surgical field. The first left SCSVC (Fig. 1A) was done with $4 \mathrm{~F}$ double lumen entral venous catheter (Arrow Guard ${ }^{\circledR}$, Arrow international Inc., USA) under Seldinger technique, using anatomical landmarks, and was removed after 4 weeks maintenance at neonatal intensive care unit (NICU) for the prevention of catheter-related infections. The bacterial culture of removed catheter tip was negative. Eight weeks after the first operation, when the body weight of the infant was $2.1 \mathrm{~kg}$, the second same left SCSVC (Fig. 1B) was performed under general anesthesia for the ventriculoperitoneal shunt (VPS) to relieve hydrocephalus. The second catheter was removed after 3 weeks maintenance at NICU and the third left SCSVC (Fig. 1C) was carried out on the same day (when the body weight was $2.31 \mathrm{~kg}$ ) under sedation. The third catheter was patent till the revision of VPS, which was done 4 weeks after the third CVC and the body weight of infant increased to $3.2 \mathrm{~kg}$.

All CVCs were done by the same anesthesiologist, with the use of maximal barriers of infection (operation gown, sterile glove, cap, mask, and large sterile sheet drape). Instead of ultrasound (US)-guided central venous catheterization, we used the classic anatomical landmark technique for the puncture of the left subclavian vein [3]. The infant was positioned supine and 15 degree of Trendelenburg position with the arms beside the body and the head turned to the right side. Landmarks for skin puncture were the clavicosternomastoid angles, formed by the lateral head of the left sternocleidomastoid muscle and the upper border of the left clavicle. Then, the needles were introduced at this point and directed inferio-medial-posteriorly at 30-45 degree to the sagittal plane. During three times for left SCSVC, we succeeded subclavian punctures with one or two skin punctures. During and after CVC procedures, there were no remarkable changes in the vital signs, pulse oximeters and electrocardiograms. The central venous catheters were advanced

Corresponding author: Hyun Soo Moon, M.D., Department of Anesthesiology and Pain Medicine, Hallym University College of Medicine, 896, Pyeongchon-dong, Dongan-gu, Anyang 431-070, Korea. Tel: 82-31-380-3945, Fax: 82-31-385-3244, E-mail: hysomoon@yahoo.co.kr

(c) This is an open-access article distributed under the terms of the Creative Commons Attribution Non-Commercial License (http:// creativecommons.org/licenses/by-nc/3.0/), which permits unrestricted non-commercial use, distribution, and reproduction in any medium, provided the original work is properly cited. 

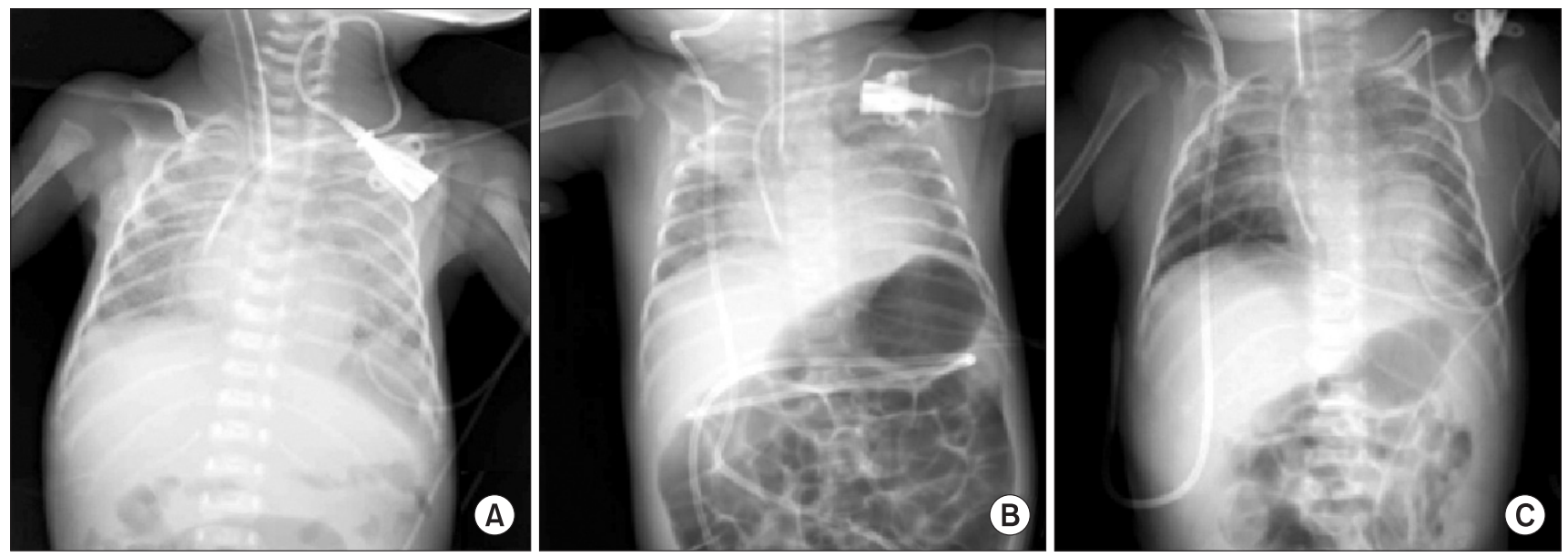

Fig. 1. Chest radiographs after (A) $1^{\text {st }}$ (B) $2^{\text {nd }}$, and (C) $3^{\text {rd }}$ supraclvicular subclavian vein catheterization. Those show catheter tips located in the right atrium.

about 6-7 $\mathrm{cm}$ from the skin and confirmed the intraatrial positioning by the follow up chest radiography.

To secure the long-term patency of the catheter, we applied intermittent low dose heparin ( $1 \mathrm{unit} / \mathrm{ml}$ ) irrigations of catheters and uses of alternative line of double lumen catheter for the infusion of TPN.

Because only the left central veins were feasible for CVC and long-term patency without any vascular complications were needed for this low birth weight premature infant, successful cannulations of the left subclavian vein were very important. US-guided central venous catheterization was introduced as the gold standard for the central vein catheterization, and has been claimed to decrease complications related to the procedure [4]. However, we did not adopt US-guided central vein catheterization, because there were not adequate anatomical spaces of sono-transducer for the left internal jugular vein due to the short neck and the large hydrocephalic head, and the left subclavian vein was not clearly visible due to the left clavicle. As such, we chose the classical Yoffa technique [3] for SCSVCs. The advantages of the supraclavicular approach over the infraclavicular access are shorter distance from the skin to vein, well defined landmarks, and fewer complications, such as pneumothorax or the great artery punctures; therefore, we applied the supraclavicular approach for three times CVC. In terms of safety, it has been known that the tip of central venous catheter should not be in the right atrium, to avoid complications. However, we advanced the catheter tips into the right atrium for long-term patency to overcome a short distance from the skin to the superior vena cava, and fortunately, intraatrial positioning did not provoke any serious complications during the three months of CVC.

The late complications related to CVC are listed as infectious, thrombotic, and embolic complications, which disturb longterm patency of catheter. To upgrade the long-term patency of catheter, low dose heparin mixed with TPN solution, and continuous infusion of low dose heparin or warfarin were known to be the effective methods [5]. For this case, we used the intermittent 1-2 $\mathrm{ml}$ heparin ( $1 \mathrm{unit} / \mathrm{ml}$ ) irrigation every 4 hours to prevent thrombosis and to keep long-term patency.

In conclusion, we performed the same left SCSVC three times for a premature infant and maintained the patency without any serious complications for about three months period. The certain knowledge of anatomical landmarks and technical skills of anesthesiologist are needed for simple, safe, and time-saving SCSVC in prematurity.

\section{References}

1. Kim TW, Ju YH, Cho HW, Lee SK, Moon HS. Central vein catheterization for prematurity. Korean J Anesthesiol 2005; 49: 72-6.

2. Karapinar B, Cura A. Complications of central venous catheterization in critically ill children. Pediatr Int 2007; 49: 593-9.

3. Yoffa D. Supraclavicular subclavian venepuncture and catheterization. Lancet 1965; 2: 614-7.

4. Rhondali O, Attof R, Combet S, Chassard D, de Queiroz Siqueira M. Ultrasound-guided subclavian vein cannulation in infants: supraclavicular approach. Paediatr Anaesth 2011; 21: 1136-41.

5. Uslu S, Ozdemir H, Comert S, Bolat F, Nuhoglu A. The effect of low-dose heparin on maintaining peripherally inserted percutaneous central venous catheters in neonates. J Perinatol 2010; 30: 794-9. 